\title{
Video Streaming Analysis in Vienna LTE System Level Simulator
}

\author{
Zakaria Ye \\ LIA/CERI \\ University of Avignon \\ 84000, Avignon, France \\ zakaria.ye@univ- \\ avignon.fr
}

\author{
Tania Jiménez \\ LIA/CERI \\ University of Avignon \\ 84000, Avignon, France \\ tania.jimenez@univ- \\ avignon.fr
}

\author{
Rachid El-Azouzi \\ LIA/CERI \\ University of Avignon \\ 84000, Avignon, France \\ rachid.elazouzi@univ- \\ avignon.fr
}

\begin{abstract}
The demand for multimedia services in mobile communication is increasing day by day due to the proliferation of end devices. To overcome the future needs of data communication on mobile devices, the $3^{\text {rd }}$ Generation Partnership Project (3GPP) has introduced a new technology which is known as Long Term Evolution (LTE) UMTS. In this paper, we study video streaming behavior on the LTE network. For this purpose, we use the Vienna LTE System Level Simulator which implements network features that correspond to our goal. We consider the parameters that may affect the users experience on a network and define ten scenarios for our simulations. We finally search for methods to fit the video streaming data traffic on the LTE network and exhibit its long range dependence.
\end{abstract}

\section{Keywords}

Video streaming, long term evolution, simulations

\section{INTRODUCTION}

Over the last few years, cellular networks have attested a rapid growth of multimedia applications, particularly video streaming applications. This growth is due to the proliferation of the end devices and the multimedia content providers like Netflix, Youtube and the video sharing platforms we can find on the social networks. The current networks, i.e., 3G and previous, suffer from this traffic load due to the bandwidth limitation which is the main constraint of the network operators. To overcome the future needs of data communication on mobiles devices, the $3^{\text {rd }}$ Generation Partnership Project (3GPP) has introduced a technology known as Long Term Evolution (LTE) of Universal Mobile Telecommunications System (UMTS). The LTE performance requirements are a high data rate and spectrum efficiency for both downlink and uplink transmission, a low latency, a high cell capacity. The major features that distinguish LTE from $3 \mathrm{G}$ are the flat-IP archtecture for the core network and the air- interface. Its main motivation is to guarantee the quality of services (QoS) of the mobile users.

Quality of Experience (QoE) has taken an important interest in the performance evaluation of multimedia traffic. Hence the satisfactory QoE to video consumers becomes a key objective for LTE system design. In this paper, we are interested to characterize properties of video streaming traffic over LTE. For this purpose, we simulate using the Vienna LTE System Level Simulator. The Vienna LTE simulator implements the different modules of the LTE technology with an abstract physical layer which catches its essential characteristics and decreases the computational level.

The first step towards defining scenarios for the streaming simulations is to understand how the streaming works: We have a video file stored in a media server. When a streaming user requests a video, this video file is divided into chunks transmitted to the user. The user holds a buffer (the playout buffer), where the video packets are stored to be played. The main component in this procedure is the base station called eNodeB in the LTE technology. The eNodeB hold a buffer for every user within its cell where the upcoming packets from the media server are stored to be transmit. Between the media server and the eNodeB, the streaming packets are transmitted smoothly. That means, in a regular manner. This is not the case for the transmission between the eNodeB and the user because of the air interface. Hence this link becomes the bottleneck for the streaming packets and introduces jitter which degrades the QoS of the streaming applications.

\section{MOTIVATIONS}

Video streaming analysis over wireless networks has been studied for many years. The oldest papers use the video streaming simulation over previous networks architecture using tools such as ns-2, OPNET or OMNET++:

In [1] authors developed a new ns- 2 module providing a RTP and RTCP implementation. A model of H.264 video streaming is developed using the OPNET modeler in [2]. Authors in [3] simulated and compared the delay, packet loss, jitter and throughput of the video streaming over an ADSL and a WiMAX networks.

There are some recent papers that deal with the video streaming over the LTE networks. Most of them focus on schedul- 
ing. In [4] the performance comparison of three scheduling algorithms is performed while authors in [5] and [6] proposed a scheduling framework for adaptive video and a quality optimized downlink scheduling respectively. The packet loss modeling is performed for the video streaming over the LTE networks in [7]. In [8] authors performed simulations using ns-3 but they did not model the video traffic, they presented users throughputs as simulation results.

To the best of our knowledge, this paper is the first attempt to address the video streaming traffic modeling since the first release of the Long Term Evolution in 2008. We decide to use the Vienna LTE Simulator that implements only LTE because it was open source and it is easy to customize it.

Our main motivation is to be able to compare theoretic models with the simulation models. These theoretic models are used in queueing theory for the computation of some metrics such as the QoS and the quality or experience (QoE).

We also study the Long Range Dependence (LRD) of the video traffic because it exhibits the bursty nature of the video traffic. This traffic is often wrongly modeled as an exponential distribution [9], the LRD shows that the interarrivals are not independent.

The rest of this paper is organized as follows: In section 3 we overview the Long Term Evolution. Section 4 presents the Vienna LTE System Level Simulator and section 5 describes the parameters of the simulations, the video traffic modeling and the results. Section 6 concludes this paper.

\section{LTE SYSTEM MODEL}

The Long Term Evolution (LTE) standard, specified by the 3rd Generation Partnership Project (3GPP) in release 8, is the next step forward in cellular $3 \mathrm{G}$ services. LTE offers significant improvements over previous technologies such as Global System for Mobile communications (GSM), Universal Mobile Telecommunications System (UMTS) and HighSpeed Packet Access (HSPA) by reforming the core network and introducing a novel physical layer. The main reasons of these changes in the Radio Access Network (RAN) system design are the need to provide higher spectral efficiency, lower delay and more multi-user flexibility than the currently deployed networks [10].

The architecture of the LTE standard is simpler than those of the previous technologies because it is based on all-IP network. There is the core network and the Radio Access Network which consists of a set of eNodeBs (Bases Stations) and Users Equipements (UEs) which communicate through the air interface. Our work is focused on the RAN layer. There is the interoperability between the LTE network and W-CDMA, GSM systems and non-3GPP systems. Then multimode UEs will support handover to and from these other systems and legacy technologies such as HSPA+ and Enhanced EDGE will continue to operate within the new data infrastructure.

As said before, the LTE introduces a novel physical layer which employs some advanced technologies that are new to cellular applications. These include Orthogonal Frequency Division Multiplexing (OFDM) and Multiple Input Multiple Output (MIMO) data transmission. In addition, the LTE physical layer uses Orthogonal Frequency Division Multiple
Access (OFDMA) on the downlink (DL) and Single CarrierFrequency Division Multiple Access (SC-FDMA) on the uplink (UL) [11].

We will just consider the downlink channel in this paper because the simulator does not implement the uplink channel but this does not affect our results.

OFDM is a modulation technique. When information is transmitted over a wireless channel, the signal can be distorted due to the multipath. Multipath is caused by signal reflection of buildings, vehicules and other obstructions. The multipath effect can cause delays between symbols which results to inter-symbol interference (ISI). OFDM eliminates ISI in the time domain in the following way: OFDM systems break the available bandwidth into many narrower subcarriers and transmit the data in parallel streams. Each subcarrier is modulated using varying levels of QAM modulation, e.g. QPSK, 16QAM, 64QAM or possibly higher orders depending on signal quality. Each OFDM symbol is therefore a linear combination of the instantaneous signals on each of the subcarriers in the channel. Also OFDM symbols are generally much longer than symbols on single carrier systems of equivalent data rate. Then OFDM symbol is preceded by a cyclic prefix (CP), which is used to effectively eliminate ISI. The sub-carriers are very tightly spaced to make efficient use of available bandwidth, yet there is no interference among adjacent sub-carriers (Inter Carrier Interference or ICI).

OFDMA is employed as the multiplexing scheme in the LTE downlink, it makes much more efficient use of network resources. Two radio frames structures are defined for the LTE: the Frequency Division Duplexing (FDD) and the Time Division Duplexing (TDD) frames of 10ms duration. Market preferences dictate that the majority of deployed systems will be FDD, so we consider LTE FDD systems only. In OFDMA, users are allocated a specific number of subcarriers for a predetermined amount of time called Transmission Time Interval (TTI) of $1 \mathrm{~ms}$. These are referred to as physical resource blocks (PRBs or RBs). Allocation of PRBs is handled by a scheduling function at the eNodeB. LTE frames are divided into 10 subframes, each subframe being $1 \mathrm{~ms}$ long. Each subframe is further divided into two slots, each of $0.5 \mathrm{~ms}$ duration. Slots consist of either 6 or 7 OFDM symbols, depending on whether the normal or extended cyclic prefix is employed. The total number of available subcarriers depends on the overall transmission bandwidth of the system. We use in our simulations a system bandwidth of 20Mhz (100 PRBs to share among the users within a sector). Each PRB is composed of 12 consecutive subcarriers for one slot (of duration $0.5 \mathrm{~ms}$ ). It is the smallest element of resource allocation assigned by the base station scheduler. OFDMA uses OFDM, one of the weakness of OFDM is the large signal peak-to-average power ratio (PAPR). This is the reason why OFDMA is not used for the uplink because one of the major challenges in cellular technologies is to reduce the power consumption for the UEs. LTE takes also advantage of the MIMO technology. MIMO enables radio systems to achieve significant performance gains by using multiple antennas at their transmitters and receivers. MIMO antennas can bring a number of potential benefits to mobile radio systems, including more reliable operation in poor signal conditions, greater spectral efficiency 
(and hence overall system capacity) and increased data rates for individual users [12]. MIMO has different modes. The spatial diversity exploits the independent fading of different signal paths between the various transmit and receive antennas to improve the reliability of a communication link. The spatial multiplexing takes advantage of multipath propagation to create a number of independent transmission channels between the transmitter and receiver, which enables two or more different signal streams to be transmitted simultaneously. The closed loop feedback and precoding enables a transmitter to take advantage of information about the transmission channel, provided by the receiver. The standard exploits the flexibility of MIMO by including the different operating modes and the system is able to switch between them to suit different operating circumstances.

With its new system architecture and novel technologies, the LTE system aims to achieve a high throughput and small latency for different services including multimedia applications. We are particularly interested in the video streaming.

\section{VIENNA LTE SYSTEM LEVEL SIMULA- TOR}

The Vienna LTE System Level Simulator of the Institute of Telecommunications at the Vienna University of Technology, is an open source software developped in MATLAB using the Object-oriented programming (OOP) capabilities that have been introduced with the release 2008a. The work has been funded by A1 Telekom Austria AG and the Christian Doppler Laboratory for Wireless Technologies for Sustainable Mobility. The simulator is available in [13]. The project first develops a link level simulator before upgrading it to a system level simulator. While link-level simulations allow for the investigation of issues such as Multiple Input Multiple Output (MIMO) gains, Adaptive Modulation and Coding (AMC) feedback, modeling of channel encoding and decoding, system level simulations focus more on network-related issues such as scheduling, mobility handling, interference management or signals propagation [14]. Then in system level simulations, the physical layer is abstracted from link level results and network performance is studied. To have an overview of the simulator, a schematic block diagram is proposed in Fig.: 1.

The core of the simulator has two parts: a link measurement model and a link performance model. The link measurement model abstracts the measured link quality to reduce the computational complexity by pregenerating a lot of parameters (eNodeBs, UEs, schedulers, resource block grids, pathloss and shadow fading maps, UEs fast fading...) before entering the main loop of the simulation. Then these parameters can be re-used during the simulation. The link quality model contains three (3) parts: (i) the macroscopic pathloss between the eNodeBs and the UEs due to the distance and the antenna gain, (ii) the shadow fading due to the obstacles between the eNodeBs and the UEs and (iii) the small-scale fading or fast fading which models the timedependant process of the channel. The pathloss, shadow fading and fast fading are combined to compute the SINR. On the other hand, the link performance model determines the BLER (Block Error Ratio) at the receiver given a certain resource allocation and Modulation and Coding Scheme (MCS). 15 different MCSs are defined, driven by 15 Channel

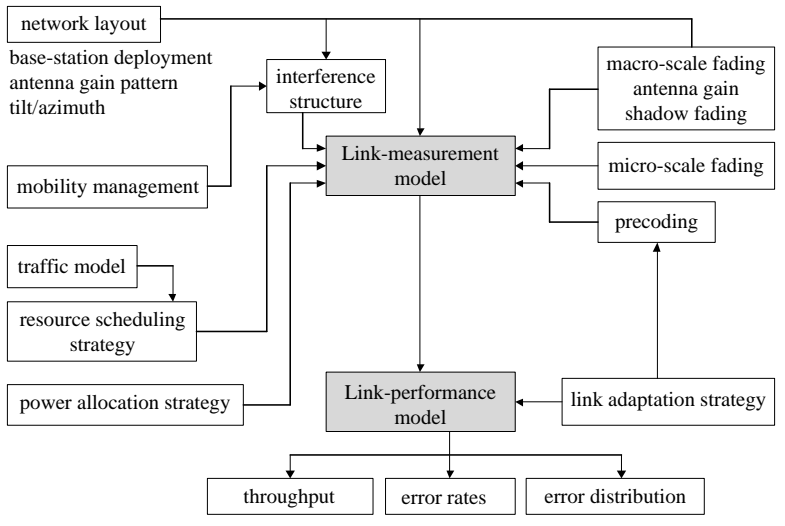

Figure 1: Schematic block diagram of the LTE system level simulator [14]

Quality Indicator (CQI) values. The CQIs use coding rates between 0.0762 and 0.9258 combined with QPSK, 16-QAM and 64-QAM modulations.

The simulator contains a main matlab file which defines the path of a simulation, it can be summarized in the pseudocode below [14]:

for each simulated TTI do

move UEs

if UE outside ROI then reallocate UE randomly in ROI

for each eNodeB do

receive UE feedback after a given feedback delay schedule users

for each UE do

1- channel state $\rightarrow$ link quality model $\rightarrow$ SINR

2- SINR, MCS $\rightarrow$ link perf. model $\rightarrow$ BLER

3- send UE feedback

The simulation is performed by defining a Region Of Interest (ROI) in which the eNodeBs and UEs are positioned and a simulation length in Transmission Time Intervals (TTIs). It is only in this area where UE movement and transmission of the Downlink Shared Channel (DLSCH) are simulated. At the programming level, the structure of the simulator is organized in modules or packages. The ChannelModel defines the downlink and uplink channels (the uplink is modeled in Vienna only for feedback reporting, no data is sending by the user), the NetworkElements module contains the eNodeBs, the UEs and also the clock which is needed for scheduling events until the end of the simulation. The scheduler module contains different types of schedulers used by the eNodeB to allocate resources to the users every TTI. The simulator contains ten (10) schedulers which can be classified in three (3) types: the Weighted Fair Queueing, the maxSNR and the Proportional Fair. The simulator contains also a WalkingModel module which defines the UEs mobility. There is also a TrafficModel for the application layer. This module contains the video traffic model, which is the scope of this paper. The other types of traffic are FTP, HTTP, VoIP, Gaming and Fullbuffer. 


\section{VIDEO STREAMING ANALYSIS}

We perform simulations using the video streaming traffic and different network parameters that affect the dynamics of the mobile communication networks.

Then we use statistical methods to fit the video data traffic using standard density functions.

\subsection{Simulation Setup}

The system model contains three (3) main components. The media server, the base station (eNodeB) and the users. Fig. 2 shows the system architecture with $N$ streaming users. The mobile users request streaming service from servers and the media streams are transmitted by the eNodeB over a fast fading channel. The streaming flow spans a wired link between the media server and the eNodeB. In this link the streams are transmitted in a regular manner. The streams span again a wireless link, between the eNodeB and the mobile user, which represents the bottleneck of the transmission because of the channel fading. Each mobile user is associated with only one flow. The system is composed of two types of buffers, the wireless buffer at the eNodeB and the playout buffer at the user side.

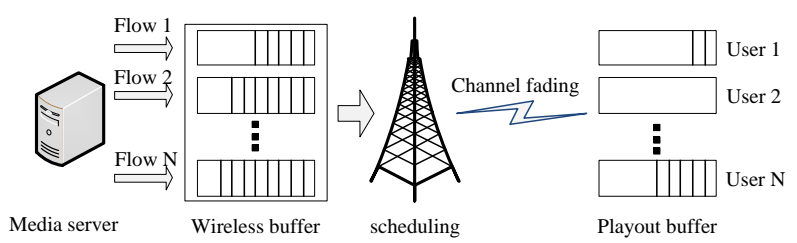

Figure 2: Media streaming system in LTE networks

The wireless buffer and the playout buffer work in a tandem way, which means that the departure process of a user in the former is exactly the arrival process in the latter. However, they work at the different time scales and at different layers. The wireless buffer works at the bit level due to the bit loading in the lower layers, and the playout buffer works at the video frame level.

The video streaming server model is integrated in the Vienna LTE simulator, in the trafficModel module. Table 1 shows the video traffic model parameters. Each video frame is splited into 8 packets (slices) where packet sizes and interarrival between packets follow a truncated Pareto distribution of parameters $\alpha$ and $K . K$ represents the minimal packet size and the minimal inter-arrival between packets. We fix the packet size to 120 bytes and the inter-arrival to $0 \mathrm{~ms}$. We assume that the media streams always have backlogged packets in the wireless buffer. This assumption holds due to two reasons: [15] First the online movies are usually very large, ranging from $100 \mathrm{MB}$ to $1 \mathrm{~GB}$. Second, most of Internet streaming servers use HTTP protocol (over TCP) to deliver streaming packets. TCP congestion control mechanism exploits the available bandwidth by pumping as more packets as possible to the wireless buffer. Therefore, we can simply regard the wireless buffers to be always saturated.

\begin{tabular}{|l|l|l|}
\hline $\begin{array}{l}\text { Information } \\
\text { types }\end{array}$ & Distribution & $\begin{array}{l}\text { Distribution } \\
\text { parameters }\end{array}$ \\
\hline $\begin{array}{l}\text { Inter-arrival time } \\
\text { between the be- } \\
\text { ginning of each } \\
\text { frame }\end{array}$ & $\begin{array}{l}\text { Deterministic } \\
\text { (based on 100fps) }\end{array}$ & $10 \mathrm{~ms}$ \\
\hline $\begin{array}{l}\text { Number of video- } \\
\text { packet in a video- } \\
\text { frame }\end{array}$ & Deterministic & 8 \\
\hline $\begin{array}{l}\text { Video-packet size } \\
\text { Truncated Pareto } \\
\text { (Max=120 bytes) }\end{array}$ & $\begin{array}{l}\mathrm{K}=120, \\
=1.2\end{array}$ \\
\hline $\begin{array}{l}\text { Inter-arrival between } \\
\text { video-packets in } \\
\text { a video-frame }\end{array}$ & $\begin{array}{l}\text { Truncated Pareto } \\
\text { Max=0ms }=0, \alpha=1.2\end{array}$ & $\mathrm{~K}=0, \alpha$ ) \\
\hline
\end{tabular}

Table 1: Video traffic model parameters

For this reason, we fix the inter-arrival between the beginning of each frame to $10 \mathrm{~ms}$. The media server sends a frame to the eNodeB every $10 \mathrm{~ms}$, that is why the inter-arrival between packets are fixed to $0 \mathrm{~ms}$ because one frame contains 8 packets. This configuration corresponds to a rate of $100 \mathrm{fps}$ on the wired link.

We consider a network with a system frequency of $2.14 \mathrm{Ghz}$ and a bandwidth of $20 M h z$. It is a macro-cells network with 7 eNodeBs. The distance between neighboring eNodeBs is $500 \mathrm{~m}$. Each eNodeB covers 3 sectors where a sector is a part of a cell. We use this topology because most of the operators use three sectors sites and by sectoring we gain better control of interference issues. As we said in section 4 , the propagation model combines the pathloss model, the shadow fading and the channel model. For the channel model, we use the Winner II+ based channel from the Winner project (one should download and put these files in the simulator before running it). It is a more complete model which covers all the propagation scenarios. The winner+ channel follows a geometry-based stochastic channel modeling approach, which allows the creation of an arbitrary double directional radio channel model [16].

The simulator has different models of pathloss: (i) the free space model, (ii) the COST231 Walfish-Ikegami model, (iii) the COST231 extended Hata model, (iv) the Vehicular Test Environment denotes by TS36942. We use these models for urban and rural environments. The shadow fading can be removed for environments without no obstacles or model by a lognormal-distributed 2D space-correlated shadow fading map with mean $\mu=0$ and standard deviation $\sigma=10$. We use also the additional_penetration_loss parameter which allows to set an additional amount of macroscopic pathloss for indoor, deep indoor, outdoor or incar simulations. The eNodeB antennas are modeled by the kathreinTSAntenna antenna pattern which has to be read from the antenna pattern file KATHREIN_antenna_files. We choose this model because it is more complete than the others. Indeed it takes into account the terrain altitude $(0 \mathrm{~m})$, the height of the eNodeB $(20 \mathrm{~m})$, the receiver height $(1.5 \mathrm{~m})$ and also the frequency of the antenna whereas the other models just depend on the antenna tilt. We use the constant user per cell distribution where the users walk in a straight line given a 


\begin{tabular}{|c|c|c|c|c|c|c|c|c|c|c|}
\hline & $\begin{array}{l}\text { pathloss } \\
\text { and } \\
\text { environ- } \\
\text { ment }\end{array}$ & $\begin{array}{l}\text { shadow } \\
\text { fading }\end{array}$ & $\begin{array}{l}\text { channel } \\
\text { model }\end{array}$ & $\begin{array}{l}\text { antenna } \\
\text { model }\end{array}$ & $\begin{array}{l}\text { number } \\
\text { of } \\
\text { users } \\
\text { (per } \\
\text { cell) }\end{array}$ & $\begin{array}{l}\text { walking } \\
\text { model }\end{array}$ & $\begin{array}{l}\text { user } \\
\text { speed } \\
(\mathrm{km} / \mathrm{h})\end{array}$ & scheduler & $\begin{array}{l}\text { trans. } \\
\text { modes }\end{array}$ & $\begin{array}{l}\mathrm{nRX} \\
\text { and } \\
\mathrm{nTX}\end{array}$ \\
\hline $\begin{array}{l}\text { scenario } \\
1\end{array}$ & $\mathrm{X}$ & claussen & winner + & kathrein & 150 & straight & 5 & alphafair & MIMO & $2 \times 2$ \\
\hline $\begin{array}{l}\text { scenario } \\
2\end{array}$ & $\begin{array}{l}\text { TS36942 } \\
\text { urban }\end{array}$ & $\mathrm{X}$ & winner + & kathrein & 150 & straight & 5 & alphafair & MIMO & $2 \times 2$ \\
\hline $\begin{array}{l}\text { scenario } \\
3\end{array}$ & $\begin{array}{l}\text { TS36942 } \\
\text { urban }\end{array}$ & claussen & $\mathrm{X}$ & kathrein & 150 & straight & 5 & alphafair & MIMO & $2 \times 2$ \\
\hline $\begin{array}{l}\text { scenario } \\
4\end{array}$ & $\begin{array}{l}\text { TS36942 } \\
\text { urban }\end{array}$ & claussen & winner + & $\mathrm{X}$ & 150 & straight & 5 & alphafair & MIMO & $2 \times 2$ \\
\hline $\begin{array}{l}\text { scenario } \\
5 \\
\end{array}$ & $\begin{array}{l}\text { TS36942 } \\
\text { urban }\end{array}$ & claussen & winner + & kathrein & $\mathrm{X}$ & straight & 5 & alphafair & MIMO & $2 \times 2$ \\
\hline $\begin{array}{l}\text { scenario } \\
6\end{array}$ & $\begin{array}{l}\text { TS36942 } \\
\text { urban }\end{array}$ & claussen & winner + & kathrein & 150 & $\mathrm{X}$ & 5 & alphafair & MIMO & $2 \times 2$ \\
\hline $\begin{array}{l}\text { scenario } \\
7\end{array}$ & $\begin{array}{l}\text { TS36942 } \\
\text { urban }\end{array}$ & claussen & winner+ & kathrein & 150 & straight & $\mathrm{X}$ & alphafair & MIMO & $2 \times 2$ \\
\hline $\begin{array}{l}\text { scenario } \\
8\end{array}$ & $\begin{array}{l}\text { TS36942 } \\
\text { urban }\end{array}$ & claussen & winner + & kathrein & 150 & straight & 5 & $\mathrm{X}$ & MIMO & $2 \times 2$ \\
\hline $\begin{array}{l}\text { scenario } \\
9\end{array}$ & $\begin{array}{l}\text { TS36942 } \\
\text { urban }\end{array}$ & claussen & winner+ & kathrein & 150 & straight & 5 & alphafair & $\mathrm{X}$ & $2 \times 2$ \\
\hline $\begin{array}{l}\text { scenario } \\
10\end{array}$ & $\begin{array}{l}\text { TS36942 } \\
\text { urban }\end{array}$ & claussen & winner + & kathrein & 150 & straight & 5 & alphafair & MIMO & $\mathrm{X}$ \\
\hline
\end{tabular}

Table 2: Simulation parameters

constant speed. When the users are out of the ROI, they are reallocated randomly in the ROI. The handover is not implemented in the simulator. But for our scenarios it is not important because we assume that the users will not leave their eNodeB during the simulation time.

The schedulers which support the video streaming traffic are alphaFair and constrained. They are variants of the proportional fair scheduler. The $\mathrm{PF}$ scheduler assigns radio resources taking into account both the experienced channel quality and the past user throughput. The goal is to maximize the total network throughput and to guarantee fairness among flows. For the PF scheduler, the metric $\omega_{i, j}$ is defined as the ratio between the instantaneous available data rate (i.e., $r_{i, j}$ ) and the average past data rate. That is, with reference to the $i$-th flow in the $j$-th sub-channel: $\omega_{i, j}=r_{i, j} / \bar{R}_{i}$ where $r_{i, j}$ is computed by the AMC module considering the CQI feedback that the UE hosting the $i$-th flow have sent for the $j$-th sub-channel; and $\bar{R}_{i}$ is the estimated average data rate. In the Vienna simulator, the av_window parameter sets the number of TTIs used to compute the average throughput $\bar{R}_{i}$. The $\alpha$ parameter controls the fairness for the alphaFair scheduler whereas the constrained scheduler uses the two parameters: $\alpha$ and $\beta$ where $\beta$ represents the priority of a UE.

The length of the simulation (in TTIs) must be set at the beginning. We do simulations of 5000 and 10000 TTIs. One weakness of the Vienna simulator is definitely the duration of the simulation. Indeed, one simulation of 10000 TTIs, 7 eNodeBs, 50 UEs per sector can take one week.

The simulator has up to four (4) transmission modes: the
Single Input Single Output (SISO) mode and the Multiple Input Multiple Output (MIMO) mode which includes the transmission diversity, the open loop and the close loop (exploits the feedback) spatial multiplexing. $n T X$ and $n R X$ denote the number of antennas of the transmitter and the receiver respectively. For the downlink, the supported antenna configurations are: $4 \times 2,2 \times 2,1 \times 2,1 \times 1$. We do simulations by varying all the parameters mentioned above. Table 2 shows the different scenarios. For each scenario, we vary one parameter while fixing the others.

\subsection{Fitting Methods}

Each eNodeB holds a buffer for every mobile user where the data is stored before the transmission. For the simulations, we consider the different types of traffic mentioned above: Video streaming, FTP, HTTP, VoIP and Gaming. These traffic types are mixed in the following way [17]: Video (20\%), FTP (10\%), HTTP (20\%), VoIP (30\%) and Gaming $(20 \%)$.

The eNodeB schedules the users within its cell every TTI. Each user is allocated a given number of resource blocks. Based on the resource blocks, the order of modulation and the coding rate, the eNodeB computes the TransportBlock size (in bits) which carry the user data.

When doing the analysis we consider only the video streaming users: At the end of each simulation, we extract the video streaming users traces from the raw simulation trace which includes all the traffic users.

The playout buffer at the mobile user stores packets transmitted over the wireless channel. The streaming packets are 
reassembled into group of pictures or "frames" according to the codec of the source.

For the traffic modelling purpose, we consider the video frames inter-arrivals, i.e. the time (in seconds) between the reception of two consecutive frames. The inter-arrival times are very important in multimedia applications. Indeed it gives an idea of the network performances and the quality of the video when playing. Then we analyse the behavior of the video traffic data using the inter-arrivals. We plot the autocorrelation function, the empirical probability density function (PDF) and the empirical cumulative distribution function (CDF) of the traffic data and we use two statistical methods: the Maximum Likelihood Estimation (MLE) and the Kalmogorov Smirnov test (KS-test) to fit the data. We work with the functions of the MATLAB statistical toolbox. The histogram bars width is computed by the Sturges's rule. It gives the best width where the range of the data is break into $k$ intervals of width $\Delta b$ each. $k$ and $\Delta b$ are basically the trial and the error and are given by

$$
k=\left[1+\log _{2}(n)\right] \quad \Delta b=\frac{\max -\min }{k}
$$

where [.] is the floor of a real number, $n$ is the number of inter-arrivals in the data, $\min$ and $\max$ are the smallest and the greatest of the inter-arrival times respectively. We plot the empirical cumulative distribution function using the Matlab stairs function. The MLE and the KS-test are used for both the empirical PDF and the empirical CDF fitting. For this purpose we consider the following twelve (12) candidate distributions: Normal, Lognormal, Gamma, Logistic, Loglogistic, Rician, Weibull, Nakagami, Extreme Value, Inverse Gaussian, t Location-Scale and Birnbaum-Saunders.

MLE is a method to estimate distribution parameters. Considering an observed sample $X_{1}, X_{2}, \ldots, X_{n}$ and a hypothesized family with probability mass function $p_{\theta}\left(x_{j}\right)=P_{\theta}(X=$ $x_{j}$ ) where $\theta$ is the unknown parameter to be estimated, the method consists of finding the value of $\theta$ that makes $L(\theta)$ as big as it can be where $L(\theta)$ is the likelihood function and is given by $p_{\theta}\left(X_{1}\right) p_{\theta}\left(X_{2}\right) \ldots p_{\theta}\left(X_{n}\right)$. The number of the estimated parameters can be one, two or even three depending on the distribution. The Matlab mle function takes as input parameters the sample data, the hypothesized distribution and a parameter $\alpha$. The value of $\alpha$ fixes the confidence interval for the estimated parameters. We set $\alpha$ to 0.01 which corresponds to $99 \%$ confidence level. The estimated parameters are used to plot the PDF and the CDF of the fitting distributions over the histograms and the empirical CDF of the users inter-arrival times.

To check how good is the fitting, we use the KalmogorovSmirnov (K-S) Test [18]. The K-S goodness-of-fit test is employed to determine the best fit among several distributions [19]. The null hypothesis $h_{0}$ implies that data samples follow a given distribution and the alternative hypothesis $h_{1}$ states the opposite. The goal of the test is to check whether to accept or reject the hypothesis $h_{0}$ and to quantify the decision. Then the approach is to examine whether the empirical distribution (empirical CDF) of a set of observations is consistent with a random sample from an assumed theoretical distribution. The estimated parameters from the mle function are used with the kstest2 which returns 0 if the fit is good enough or 1 otherwise.
We conclude that Rician and Logistic distributions fit the data better than the others.

The autocorrelation function measures the dependence between two inter-arrival times. It tests whether the data exhibit long-range dependence or not. We use the Matlab autocorr function to plot the autocorrelation function of the inter-arrival times.

\subsection{Simulation Results}

Figures 3 and 4 show the cumulative distribution function and the probability density function of the video streaming inter-arrival times, i.e., the time between the reception of two consecutive frames where the arriving time of a frame is the arriving time of the last packet of this frame. The inter-arrival time is called the jitter which is an important metric of the quality of service (QoS). A large jitter involves a bad QoS. For the video streaming applications, the media player has a deterministic rate which is the number of frames displayed per second (fps). There exist three main frame rate standards, $24 \mathrm{fps}, 25 \mathrm{fps}$ and $30 \mathrm{fps}$. For a rate of $25 \mathrm{fps}$, a frame is displayed every $40 \mathrm{~ms}$. We can see on figure 4 that most of the inter-arrival times lay in the interval $10 \mathrm{~ms}-20 \mathrm{~ms}$. This scenario corresponds to 150 users within a cell of diameter $500 \mathrm{~m}$.

These results confirm not only the performances of the LTE technology but also their importance for network provisioning, predicting utilization of network resources, and for planning network developments.

The fitting distributions are also an important result because we need to use theoretical models for research purpose. Often it is difficult to work directly with the traffic traces of the content providers because they are not always accessible for confidentiality purpose; or simply because it is not practical. Then we develop mathematical models based on traditional queuing theory to compute streaming relative metrics such as the quality of service and the quality of experience.

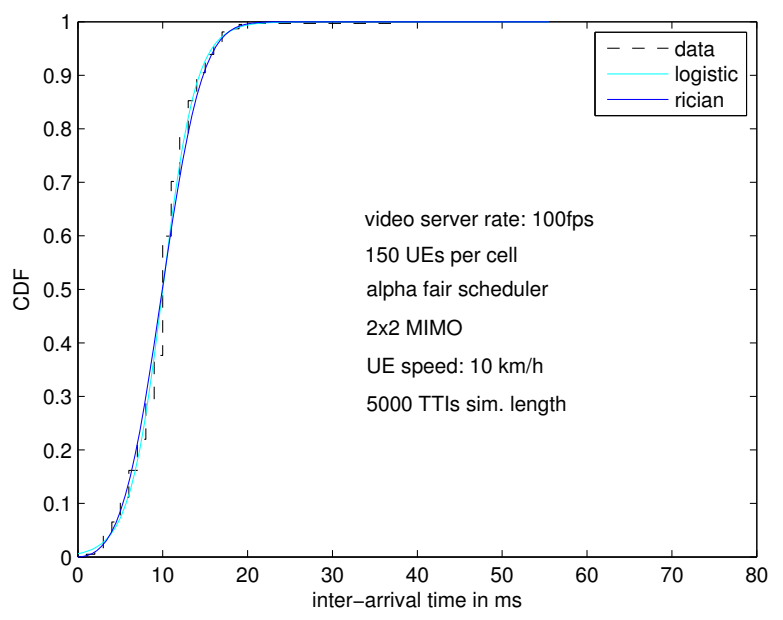

Figure 3: Cumulative distribution function of the inter-arrival times 


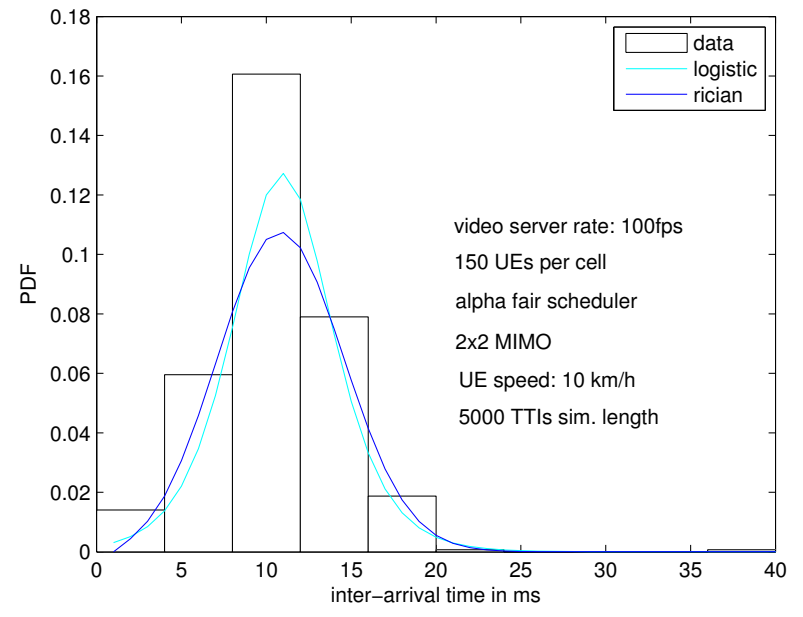

Figure 4: Probability density function of the interarrival times

Figure 5 shows the inter-arrival times autocorrelation func-

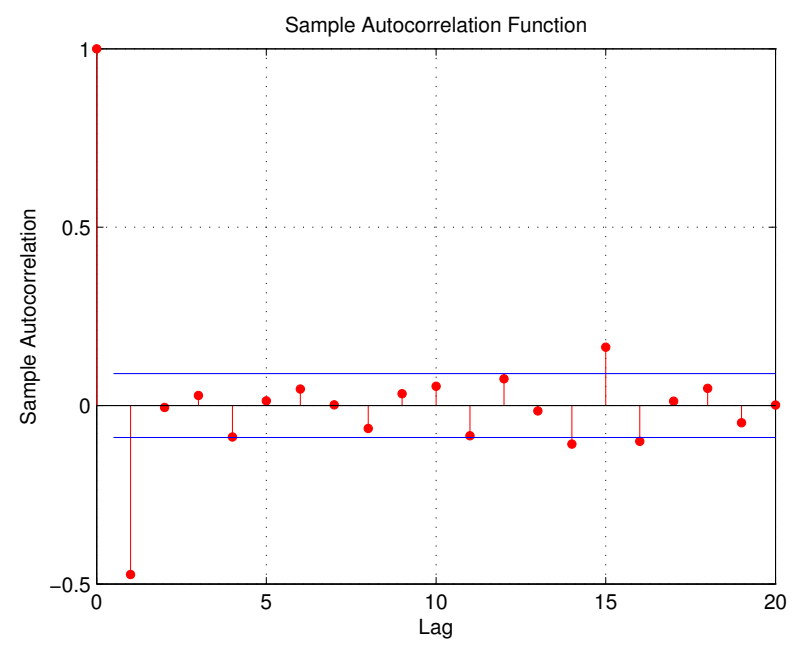

Figure 5: Interarrival times autocorrelation function

tion. It computes the autocorrelation coefficients and plots them as a function of the lag. If the data are independent (uncorrelated) then the correlations should be near zero for all lags. We can see in the figure that the coefficients are not close to zero for many lags, that exhibit the long range dependence of the video streaming inter-arrival times.

\section{CONCLUSIONS}

In this paper, we consider the video streaming applications over the Long Term Evolution (LTE) networks. Our purpose is to analyze the video traffic data through the dynamics of the LTE networks. We use the Vienna LTE System Level Simulator for our study. Simulations were running with different values for the parameters: the pathloss, the users speed, the network layout, the transmission modes.
The video server was integrated to the simulator. Other types of traffic were also present. We process the simulation traces: For this purpose we consider the inter-arrival times of the streaming users because they are very important in the multimedia applications. They give an idea about the performances of the network. The best representation of the data is their distributions, i.e., the probability distribution function and the cumulative distribution function. We use statistical methods like Maximum Likelihood Estimation and the Kalmogorov Smirnov test to fit the traffic data. We plot the PDF, the CDF and the fitting distributions using some functions of the statistical toolbox of MATLAB. We also plot the autocorrelation function which shows the long range dependance of the inter-arrival times.

From the plots, we can see that most of the inter-arrival times are in the interval $10 \mathrm{~ms}-20 \mathrm{~ms}$. Knowing that the mean service time of the video playbacks is $40 \mathrm{~ms}$, that confirms the high performance of the LTE networks.

Our results can be used to analyse the quality of experience of the video streaming applications and the quality of service of the LTE networks.

As future works, we plan to do the same simulations with ns-3, in order to compare the performances of the two simulators.

\section{ACKNOWLEDGMENT}

This work has been carried out in the framework of IDEFIX project, funded by the ANR under the contract number ANR-13-INFR-0006.

\section{REFERENCES}

[1] Mario Montagud, Fernando Boronat, and Vicent Vidal. Simulation platform for video streaming evaluation. pages 397-401, sept. 2010.

[2] Doggen Jeroen and Van der Schueren Filip. Design and simulation of a h.264 avc video streaming model. March 2008.

[3] Ahmed Bilal and Arshad M. Junaid. Simulation and comparative analysis of video streaming over adsl \& wimax network.

[4] Biswapratapsingh Sahoo. Performance comparison of packet scheduling algorithms for video traffic in LTE cellular network. IJMNCT, 3, June 2013.

[5] Jiasi Chen, Rajesh Mahindra, Mohammad Amir Khojastepour, Sampath Rangarajan, and Mung Chiang. A scheduling framework for adaptive video delivery over cellular networks. pages 389-400. ACM, 2013.

[6] Xiaolin Cheng and Prasant Mohapatra. Quality-optimized downlink scheduling for video streaming applications in lte networks. In GLOBECOM, pages 1914-1919. IEEE, 2012.

[7] Moustafa M Nasralla, CTER Hewage, and Maria G Martini. Subjective and objective evaluation and packet loss modeling for 3d video transmission over lte networks. In Telecommunications and Multimedia (TEMU), International Conference on, pages 254-259. IEEE, 2014.

[8] Abdurrahman Fouda and al. Real-time video streaming over ns3-based emulated lte networksk. IJECCT, 4, May 2014.

[9] Yuedong Xu, Eitan Altman, Rachid El-Azouzi, Majed Haddad, Salah-Eddine Elayoubi, and Tania Jimenez. Analysis of Buffer Starvation With Application to Objective QoE Optimization of Streaming Services. IEEE Transactions on Multimedia, 16(3):813-827, 2014. 
[10] E. Dahlman, S. Parkvall, J. Skold, and P. Beming. $3 G$ Evolution: HSDPA and LTE for Mobile Broadband. Academic Press, 2th edition, 2007.

[11] J. Zyren and Dr. W. McCoy. Overview of the 3GPP Long Term Evolution Physical Layer, July 2007.

[12] http://www.unwiredinsight.com/2013/lte-mimo.

[13] https://www.nt.tuwien.ac.at/downloads/featureddownloads/.

[14] J. C. Ikuno, M. Wrulich, and M. Rupp. System level simulation of Lte networks. IEEE VTC2010-Spring, May 2010.

[15] Y. Xu, E. Altman, R. El-Azouzi, S. Elayoubi, and M. Haddad. Qoe Analysis of Media Streaming in Wireless Data Networks. Springer Berlin Heidelberg, 2:343-354, May 2012.

[16] http://www.ist-winner.org/phase_2_model.html.

[17] IEEE Working Group 802.20 Permanent Document. Traffic Models for IEEE 802.20 MBWA System Simulations, July 2003.

[18] Bozidar Vujicic, Nikola Cackov, Svetlana Vujicic, and Ljiljana Trajkovic. Modeling and characterization of traffic in public safety wireless networks. In In Proc. of SPECTS, pages 214-223, 2005.

[19] Ralph B D'Agostino and Michael A Stephens, editors. Goodness-of-fit Techniques. Marcel Dekker, Inc., New York, NY, USA, 1986. 